\title{
Calidad y prestigio de la edición académica. El caso colombiano*
}

\author{
Quality and Prestige of Scholarly Publications. The Colombian Case \\ Qualidade e prestígio da edição acadêmica. O caso colombiano
}

Elea Giménez Toledo ${ }^{\text {a }}$

DOI: https://doi.org/10.11144/Javeriana.syp.40-78.cpea

Consejo Superior de Investigaciones Científicas, España

elea.gimenez@cchs.csic.es

ORCID: https://orcid.org/0000-0001-5425-0003

Recibido: 27 Julio 2020

Juan Felipe Córdoba Restrepo

Aceptado: 30 Marzo 2021

Universidad del Rosario, Colombia

Publicado: 30 Junio 2021

ORCID: https://orcid.org/000-0001-9024-9309

Esteban Giraldo González

Universidad Santo Tomás, Colombia

Universidad Carlos Ill de Madrid, España

ORCID: https://orcid.org/0000-0002-1496-1668

Jorge Mañana-Rodríguez

Consejo Superior de Investigaciones Cientificas, España

ORCID: https://orcid.org/0000-0002-0717-5271

\section{Resumen:}

Reconocer la publicación de libros académicos en español en los procesos de evaluación científica requiere disponer de información objetiva y precisa sobre libros, colecciones y editoriales. En este trabajo, se presenta un estudio sobre el prestigio de las editoriales académicas - colombianas y no colombianas - a partir de una encuesta realizada entre profesores e investigadores colombianos, siguiendo el modelo del proyecto SPI. La encuesta obtuvo una tasa de respuesta del $17 \%$. El estudio permite identificar los núcleos de editoriales académicas relevantes para los investigadores, tanto del ámbito nacional como internacional. Un total de 167 editoriales colombianas y 345 no colombianas fueron reconocidas por los académicos. El indicador ICEE genera una lista ordenada de editoriales. Los resultados muestran cómo se concentra el prestigio en pequeños núcleos, liderados por la edición universitaria. Atendiendo al origen de las editoriales extranjeras, se identifican dos "polos" de influencia: el del español/iberoamericano, en el que destacan las editoriales españolas, mexicanas y argentinas y el del inglés/anglosajón, representado por Estados Unidos, Reino Unido y Holanda. La calidad de las obras publicadas, la trayectoria de la editorial, su especialización y el aval científico de sus directores son las variables con mayor peso en la determinación del prestigio. En la discusión, se sitúan estos resultados en un contexto más amplio de evaluación científica, bibliodiversidad y multilingüismo en la comunicación científica.

Palabras clave: libros académicos, editoriales académicas, evaluación científica, indicadores de prestigio, Humanidades y Ciencias Sociales.

\section{Abstract:}

Acknowledgment of scholarly book publishing in Spanish in the scientific evaluation processes requires objective and accurate information about the books, collections and publishing houses. This work presents a study on the prestige of - both Colombian and non-Colombian - scholarly publishing houses based on a survey using the SPI-project model and answered by Colombian teachers and researchers. The survey answer rate was $17 \%$. This study identified the publishing hotspots relevant for the researchers both in the national and international spheres. In total 167 Colombian and 345 non Colombian publishing houses were recognized by the scholars. The ICEE indicator provides an ordered list of publishing houses. The results show how the prestige is concentrated in small hotspots led by college publications. Regarding the national origin, the foreign publishing houses are grouped in two influential "origins": the Spanish/Ibero-American group with prominent Spanish, Mexican and Argentinian publishing houses and the English/Anglo-Saxon group including American, British and Dutch publishing houses. The quality of the publication, the publisher track record, the specialized fields and the scientific support by the directors are the most important variables when

Notas de autor

\footnotetext{
a Autora de correspondencia. Correo electrónico: elea.gimenez@cchs.csic.es
} 
determining the prestige. In the Discussion these results are considered in a broader context of scientific evaluation, bibliodiversity and multilingualism typical to the scientific communication.

Keywords: scholarly books, scholarly publishing houses, scientific evaluation, prestige indicators, Humanities and Social Sciences.

\section{Resumo:}

Reconhecer a publicação de livros acadêmicos em espanhol nos processos de avaliação científica exige ter informações objetivas e precisas sobre livros, coleções e editoras. Neste artigo é apresentado um estudo sobre o prestígio das editoras acadêmicas colombianas e não colombianas - a partir de inquérito realizado entre professores e pesquisadores colombianos, seguindo o modelo do projeto SPI. O inquérito obteve uma taxa de resposta de $17 \%$. O estudo permite identificar os núcleos das editoras acadêmicas relevantes para os pesquisadores, tanto nacional quanto internacionalmente. Um total de 167 editoras colombianas e 345 não colombianas foram reconhecidas pelos acadêmicos. O indicador ICEE gera uma lista ordenada de editoriais. Os resultados mostram como o prestígio se concentra em pequenos núcleos, liderados pela edição universitária. Considerando a origem das editoras estrangeiras, identificam-se dois "polos" de influência: a espanhola/ibero-americana, em que destacam-se as editoras espanholas, mexicanas e argentinas, e a inglesa/anglo-saxônica, representada pelos Estados Unidos, o Reino Unido e os Países Baixos. A qualidade das obras publicadas, a história da editora, a sua especialização e o aval científico dos seus dirigentes são as variáveis com maior peso na determinação do prestígio. Na discussão, esses resultados são colocados em um contexto mais amplo de avaliação científica, bibliodiversidade e multilinguismo na comunicação científica.

Palavras-chave: livros acadêmicos, editoriais acadêmicas, avaliação científica, indicadores de prestígio, Humanidades e Ciências Sociais.

\section{Introducción}

Rara vez se mencionan los libros académicos como indicadores del desempeño en la actividad científica en los análisis macro que se realizan y publicitan a nivel de país. Los artículos de revistas científicas cubiertos por las bases de datos Web of Science (Clarivate Analytics) y Scopus (Elsevier) constituyen la referencia para medir la producción científica de un país y permitir su comparabilidad internacional. Sin embargo, la producción científica es mucho más que ese conjunto de artículos. Si bien los libros y capítulos de libros son considerados en algunos casos, en la evaluación de investigadores de estas disciplinas (nivel micro), generalmente tienen un peso menor que los artículos de revistas científicas indexadas. La evaluación de la actividad científica debe considerar todos los tipos de resultados de investigación que puede generar una comunidad científica. Así lo reivindica el equipo de trabajo de la acción COST European Network for Research Evaluation in the Social Sciences and the Humanities (ENRESSH, 2017), entre otros.

Excluir o minusvalorar los libros académicos en los procesos de evaluación de las Humanidades y las Ciencias Sociales, así como en otras disciplinas como la Administración o la Economía, es un contrasentido, teniendo en cuenta que son precisamente los libros canales de comunicación más utilizados, citados y reconocidos por la comunidad científica que integra estas áreas. Además, resulta paradójico en el entorno iberoamericano, considerando que algunos países como México, España, Brasil, Argentina o Colombia son grandes potencias editoriales, con una especial dedicación al libro académico.

En 2012, se realizó la primera encuesta sobre el prestigio de las editoriales académicas (Giménez-Toledo et al., 2012), desarrollada por el grupo de Investigación sobre el Libro Académico (ILIA) del Consejo Superior de Investigaciones Científicas de España. La consulta se dirigió a más de 11.000 humanistas y científicos sociales españoles y obtuvo una tasa de respuesta del $26 \%$. Tuvo como objetivo identificar aquellas editoriales españolas y extranjeras relevantes para el trabajo académico de los investigadores en cada una de las disciplinas. Al hacerlo, no solo se delimitaron las editoriales de perfil académico y/o relevantes para la investigación, sino que pudieron identificarse aquellas editoriales que resultan más prestigiosas y aquellas que gozan de menos reconocimiento, por distintos tipos de razones. Los listados ordenados de editoriales fueron el primer resultado publicado en el sistema de información de editoriales Scholarly Publishers Indicators (SPI, 2018), que han ido actualizándose mediante la realización de nuevas encuestas en 2014 y 2018. Además, el sistema ha ido incorporando otras informaciones como los procedimientos de selección de originales seguidos por 
las editoriales, sus perfiles de especialización temática y su presencia o ausencia en sistemas de información internacionales (SPI, 2018).

Los datos e indicadores contenidos en SPI se han ido completando y analizando en diferentes informes para obtener visiones panorámicas de la edición de libros académicos en España (Giménez Toledo, 2017, 2018, 2019). Los indicadores de prestigio y sistemas de selección de originales son tenidos en cuenta por la Agencia Nacional de Evaluación de la Calidad y la Acreditación (ANECA) y otras agencias autonómicas de evaluación de la actividad investigadora en España (ANECA, 2018; Comisión Nacional Evaluadora de la Actividad Investigadora [CNEAI], 2019).

Con respecto a la evaluación de la producción de libros académicos de las editoriales colombianas, existe el consenso de la necesidad de crear metodologías que puedan ser reconocidas por todos los actores del sistema de ciencia y tecnología nacional (Uribe Tirado, 2019). Es conocido, además, que las convocatorias para registro y reconocimiento de las editoriales científicas llevadas a cabo por Colciencias entre 2012 y 2013 (Colciencias, 2012, 2013) no continuaron en el tiempo. En esa medida, los resultados de esas convocatorias no permiten establecer criterios a partir de los cuales hacer análisis ni comparaciones fiables para el país.

Disponer de información sobre la edición de libros académicos permite mostrar la relevancia de este tipo de producción científica en distintas disciplinas, especialmente las de Humanidades y Ciencias Sociales. De este modo, se reivindica la consideración del libro académico como un resultado relevante de la investigación en estas áreas. Además, la investigación realizada permite contar con indicadores objetivos sobre la edición académica, facilitando así los procesos de evaluación de la actividad investigadora, por cuanto constituyen una información en la que basar algunas decisiones. Estos indicadores, asimismo, salvan la falta de cobertura de editoriales académicas del ámbito no anglosajón en las bases de datos internacionales como Book Citation Index y Scopus (Leydesdorff y Felt, 2012). Desarrollar fuentes completas de datos sobre la edición de libros académicos en español es una tarea relevante para hacer visible y reconocida la actividad científica de los investigadores que escriben sobre sus resultados de investigación, así como la de los editores que transforman estos textos en libros.

Por otra parte, merece la pena ir desentrañando las claves de la falta de circulación o reconocimiento de las editoriales académicas iberoamericanas entre las comunidades académicas hablantes de español o portugués. En los estudios mencionados más arriba (SPI), se ha podido observar la baja frecuencia con que las editoriales universitarias latinoamericanas y españolas eran mencionadas por los investigadores españoles, variando entre un $6,4 \%$ y un 32,6 \% entre las distintas disciplinas. Esta observación no concuerda con los datos de producción editorial. Se producen muchos libros académicos en español que luego son poco reconocidos por la comunidad académica iberoamericana. El $13 \%$ de toda la producción editorial en América Latina se debe a las editoriales universitarias (Cerlalc, 2019). En Colombia, la producción editorial universitaria representa el $29 \%$ del total según el informe de Cerlalc (2019) y un 27,7 \% según la agencia ISBN colombiana (Asociación de Editoriales Universitarias de Colombia [ASEUC], 2019). En España, la edición académica - contabilizando aquí la universitaria y la privada/comercial — representa el $17 \%$, según los últimos datos disponibles (Giménez Toledo, 2017).

\section{Objetivos}

Las distintas razones aportadas hacían apropiado llevar a cabo una consulta sobre la edición académica dirigida a investigadores colombianos, similar a la desarrollada en España en años anteriores. Los objetivos de esta consulta se concretan en:

a) Delimitar el sector editorial académico colombiano, identificando el conjunto de editoriales que es reconocido por la academia. 
b) Identificar las editoriales más prestigiosas, tanto colombianas como no colombianas, de acuerdo con el criterio de los académicos colombianos.

c) Analizar la tipología y el origen geográfico de las editoriales académicas más prestigiosas.

d) Realizar un análisis comparativo con los resultados de las encuestas en España para identificar algunas cuestiones relacionadas con la circulación y el reconocimiento del libro en español.

\section{Metodología}

Entre el 24 de octubre de 2018 y el 14 de marzo de 2019, se remitió un correo electrónico (anexo 1) a 2426 académicos colombianos, solicitando su colaboración con el proyecto mediante la cumplimentación de un cuestionario en línea (anexo 2). Se obtuvieron 408 respuestas utilizables (tasa de respuesta: 16,81\%). Los destinatarios de la encuesta fueron profesores e investigadores en Ciencias Humanas y Sociales de las universidades colombianas, con trayectorias consolidadas, identificados por las vicerrectorías y unidades de investigación de las 67 universidades que en ese momento hacían parte de la ASEUC, que ha actuado como parte imprescindible de este estudio y coordinadora de la relación institucional con universidades.

El cuestionario en línea se desarrolló utilizando la herramienta Google Forms y las respuestas fueron recolectadas a través de hojas de cálculo de Google. En la encuesta, se solicitaba a los académicos tres cuestiones básicas: a) señalar las diez editoriales colombianas más prestigiosas, b) señalar las diez editoriales no colombianas más prestigiosas y c) señalar las cinco colecciones académicas de editoriales colombianas más prestigiosas. En el caso de las preguntas relacionadas con el prestigio de las editoriales, también se les preguntó si habían sido autores de las editoriales mencionadas o no y, en el caso de las colecciones académicas, a qué editorial pertenecía la colección. Por otra parte, también se les solicitó que señalasen los elementos que tenían en cuenta a la hora de valorar el prestigio de una editorial, a partir de una lista desplegable (con opción de respuesta múltiple y abierta). La estructura del cuestionario implica que los participantes ordenan las editoriales de acuerdo con el prestigio percibido, situando en primer lugar la editorial más prestigiosa, en segundo la que le sigue en prestigio y así sucesivamente.

Tras cuatro recordatorios dirigidos a los académicos y encaminados a incrementar la tasa de respuesta, se dio por finalizada la fase de consulta y se procedió al análisis de los datos. Como en los estudios anteriores realizados en España (grupo ILIA del CSIC), se trabajó intensivamente en la normalización de la nomenclatura de las editoriales. Pasado el proceso de depuración de los datos se aplicó el mismo indicador utilizado en el ranking de prestigio de editoriales utilizado en SPI (Scholarly Publishers Indicators): el Indicador de Calidad de Editoriales según los Expertos (ICEE).

El ICEE para una determinada editorial es $=\mathrm{A}^{*} 10+\mathrm{B}^{*} 9+\mathrm{C}^{*} 8+\mathrm{D}^{*} 7 \ldots+\mathrm{J}^{*} 1$

donde:

A es el número recibido de votos/menciones por la editorial como la más prestigiosa, $\mathrm{B}$ es el número de votos recibidos por la editorial como la segunda más prestigiosa, $\mathrm{C}$ es el número de votos recibidos por la editorial como la tercera más prestigiosa, etcétera.

La distribución de las respuestas por área de conocimiento fue muy desigual, lo que impide obtener una visión sólida del prestigio de las editoriales por disciplinas. Sin embargo, y a pesar de que la tasa de respuesta es considerablemente más baja que en los estudios previos, sí puede obtenerse la visión de conjunto en cuanto al núcleo de editoriales académicas más relevantes — globalmente- para la comunidad académica. En la medida en que no es posible presentar un listado cerrado de colecciones discriminado por editoriales, la gran variedad de colecciones indicadas por los investigadores no permite un análisis lo suficientemente preciso del prestigio de las mismas. Este hecho sugiere que la noción de colección en Colombia no tiene una relevancia significativa ni para los autores ni para el grueso de los editores universitarios, lo que invita al análisis en estudios futuros. 
Los valores del indicador se calcularon para las editoriales colombianas y no colombianas de tres formas diferentes, considerando: el total de respuestas, solo las de los académicos que no han sido autores de esa editorial y solo las de aquellos que sí han sido autores con una editorial. Este tratamiento de la información tiene como objetivo determinar el peso de los posibles sesgos en la valoración de una editorial cuando se es autor.

\section{Resultados}

Un total de 167 editoriales colombianas y 345 no colombianas fueron señaladas en la consulta. Las siguientes tablas reflejan las 20 editoriales más prestigiosas, tanto colombianas como no colombianas, de acuerdo con el indicador ICEE calculado a partir del total de respuestas. Los listados completos de editoriales, así como su desagregación en función de si los académicos eran o no autores de la editorial, pueden consultarse en SPI Colombia (2019).

TABLA 1.

Editoriales más prestigiosas, colombianas y no colombianas

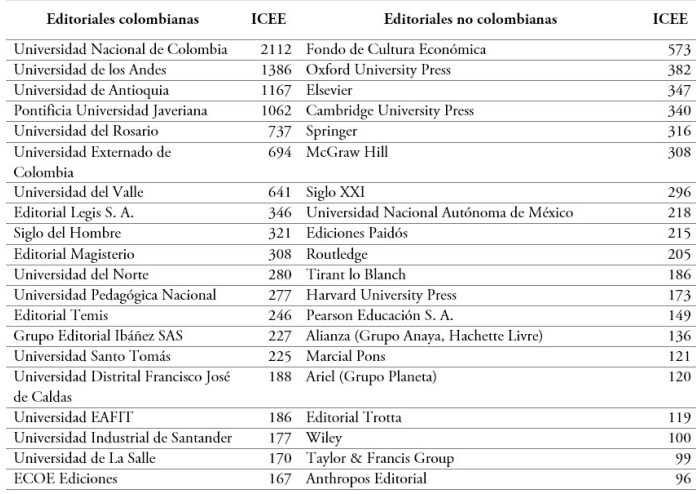

Fuente: elaboración propia

Es relevante señalar que la mayoría de las editoriales colombianas más prestigiosas son universitarias, mientras que, en el listado de editoriales no colombianas, no son las universitarias las que predominan (tabla 2). Esto está relacionado con la calidad de la edición y también con la propia conformación del sector editorial académico en Colombia, en el que predomina la edición universitaria y en el que hay pocos sellos privados/ comerciales. Las editoriales académicas no universitarias no tuvieron como objetivo servir de canal para la divulgación de textos académicos en el formato libro, lo que llevó, con el tiempo, a que las universidades decidieran desarrollar sellos editoriales para garantizar la publicación de materiales, que sin estos sellos hubieran quedado sin publicar.

TABLA 2.

Tipología de editoriales citadas

\begin{tabular}{lcccc}
\hline Tipo de editorial & $\begin{array}{c}\text { Frecuencia } \\
\text { (editoriales } \\
\text { colombianas) }\end{array}$ & $\%$ & $\begin{array}{c}\text { Frecuencia } \\
\text { (Editoriales no } \\
\text { colombianas) }\end{array}$ & $\%$ \\
\hline $\begin{array}{l}\text { Editorial } \\
\text { privada/comercial }\end{array}$ & 79 & 47,31 & 236 & 68,41 \\
\hline $\begin{array}{l}\text { Editorial } \\
\text { universitaria }\end{array}$ & 60 & 35,93 & 74 & 21,45 \\
\hline $\begin{array}{l}\text { Editional sector } \\
\text { público }\end{array}$ & 28 & 16,77 & 35 & 10,14 \\
\hline
\end{tabular}


Para el mismo conjunto de editoriales, se quiso observar la diferencia de percepción entre autores y no autores de cada editorial. Con ello puede estimarse hasta qué punto el ser o no autor de la editorial interviene en su percepción y, por tanto, qué sesgos podrían darse (Giménez Toledo, 2018). De manera genérica, se puede afirmar que el hecho de ser autor de una editorial puede producir cambios en la percepción, tanto en sentido positivo - la editorial resultaría mejor valorada - como en sentido negativo - la editorial resultaría peor valorada-. En el análisis de datos realizado, se observa que hay editoriales prestigiosas que resultan peor valoradas cuando se trata solo la opinión de los autores y, al contrario, editoriales que se posicionan mejor si se considera la opinión que quienes han publicado en ellas. No se trata aquí de ofrecer datos pormenorizados de lo que sucede en cada editorial sino, más bien, de constatar que los cambios se producen en los dos sentidos y no solo en el positivo, crítica que inicialmente se formulaba en relación con los resultados de SPI (Giménez Toledo, 2018).

El análisis de los países de origen de las editoriales que aparecen reconocidas entre las no colombianas proporciona una idea de las influencias y de la circulación de ideas en la academia colombiana (tabla 3). Pero, además, estos datos pueden resultar en cierto modo informativos de la actividad comercial, la distribución y el marketing de las editoriales extranjeras en Colombia. La relación de editoriales que se obtiene como resultado no solo muestra lo que se reconoce como prestigioso, sino también lo que se conoce, las editoriales que hacen llegar su producción a las librerías y bibliotecas del país.

TABLA 3.

Países de origen de las editoriales no colombianas citadas en el estudio

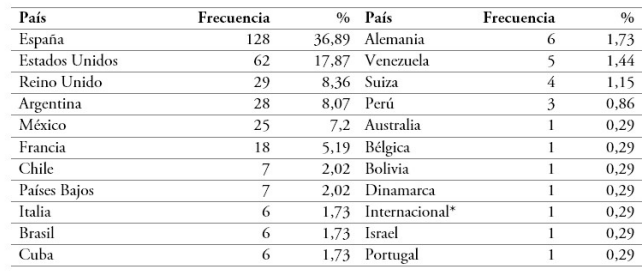

* Se agrupan bajo este epígrafe las editoriales multinacionales, con sede en varios países, que han sido mencionadas por los académicos. Fuente: elaboración propia

La lista está encabezada por España, seguida a una considerable distancia por Estados Unidos, lo que podría interpretarse en dos sentidos. El primero relacionado con la disponibilidad y accesibilidad (tecnológica y económica) de los libros de editoriales españolas en Colombia; el segundo, con el contenido de las obras: el idioma, los temas y los enfoques pueden ser razones subyacentes a la receptividad de la academia colombiana hacia obras publicadas por editoriales españolas.

Si se agregan los valores del indicador de prestigio por países, se obtiene una visión complementaria a la tabla anterior (tabla 4). Estos valores muestran la existencia de dos polos de influencia o de reconocimiento: el polo del español y de la cultura iberoamericana, representado por editoriales españolas, mexicanas y argentinas y el polo del inglés y de la cultura anglosajona, por editoriales estadounidenses, británicas y holandesas. 
TABLA 4.

Prestigio acumulado bruto y porcentaje del prestigio total acumulado por las editoriales de los diferentes países

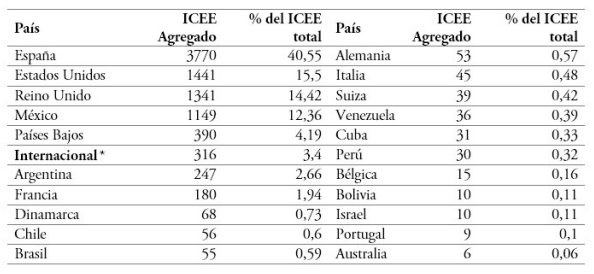

* Se agrupan bajo este epígrafe las editoriales multinacionales, con sede en varios países, que han sido mencionadas por los académicos. Fuente: elaboración propia

Al final del cuestionario, se incluía una pregunta relativa a las razones que conforman el prestigio de una editorial, lo que ayuda a entender qué significado tiene el concepto "prestigio" para un investigador o profesor. La figura 1 muestra la relación de componentes que conforman el prestigio, a partir de las respuestas de los académicos. Además, aparecen ordenadas de mayor a menor frecuencia.

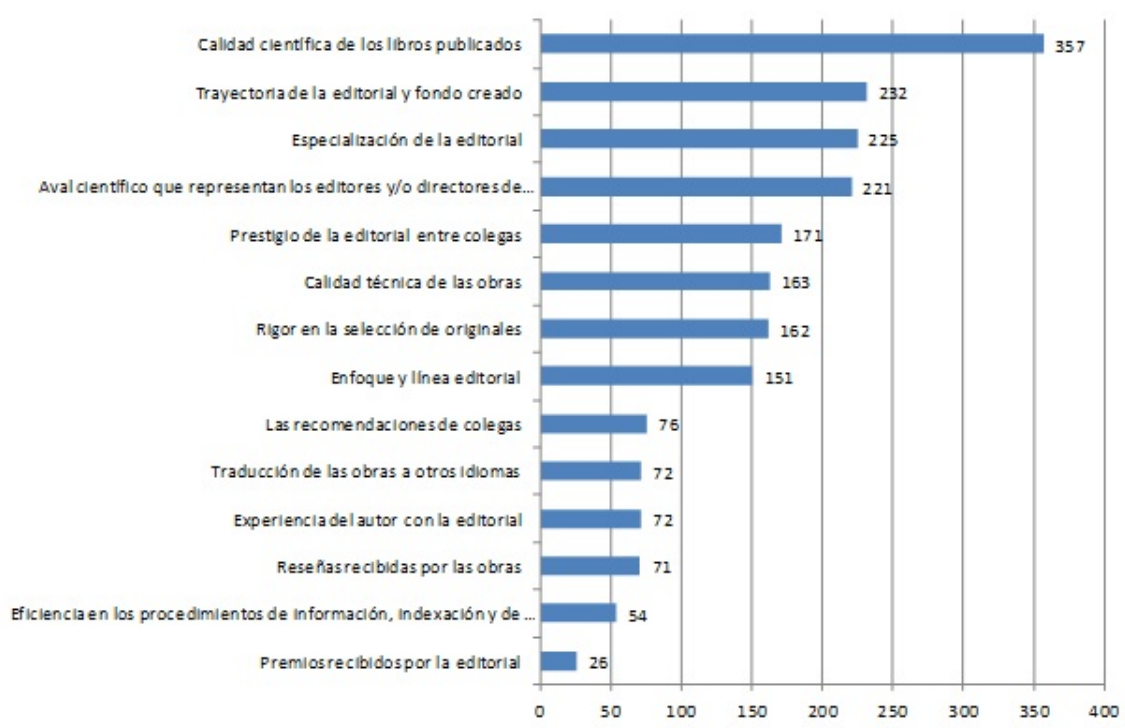

FIGURA 1.

Componente del concepto "prestigio" de la editorial

Fuente: elaboración propia

No cabe duda de que es la calidad de los libros que publica una editorial lo que subyace, de manera principal, al concepto de prestigio de una editorial. Esto, además, guarda una relación estrecha con las dos características que le siguen en frecuencia: el catálogo de la editorial y su especialización. Las tres características son comunes a colecciones o editoriales que se convierten en referencia para las distintas especialidades científicas. Esto sugiere que, si bien hay otras características que intervienen en la determinación del prestigio, son aquellas relacionadas con los contenidos publicados las que tienen un mayor peso a la hora de valorar el prestigio de una editorial. 


\section{Conclusiones y discusión}

La tasa de respuesta de este estudio ha sido menor que en otras consultas similares (Giménez-Toledo et al., 2012), lo que implica que los resultados no pueden tomarse como estadísticamente representativos, pero sí suficientes para obtener una primera aproximación a la percepción del prestigio de las editoriales por parte de los académicos colombianos, así como al panorama nacional de la edición académica en Colombia. De hecho, los resultados permiten identificar los núcleos básicos de editoriales relevantes, incluyendo a aquellas editoriales privadas que están publicando libros académicos. En Colombia, representan un sector minoritario, lo que contrasta con el panorama español en el que la edición privada en Humanidades y Ciencias Sociales representa un $85 \%$ frente al $15 \%$ de la universitaria. También permiten observar la bibliodiversidad existente: un total de 167 editoriales colombianas han sido reconocidas por los investigadores, aunque el prestigio se concentra en unas pocas, lo que, por una parte, es natural y por otra, consistente con lo que ocurre en otros escenarios editoriales. Ambos datos - bibliodiversidad y limitación del prestigio en unos cuantos sellos editoriales - invitan a pensar en que se están publicando libros académicos que cubren temas y enfoques diversos, probablemente apegados a problemas locales y regionales y publicados fundamentalmente en español. Esto está directamente relacionado con las reivindicaciones de la Iniciativa Helsinki sobre multilingüismo en la comunicación científica (Federación de Asociaciones Científicas Finlandesas, Comité para la Información Pública, Asociación Finlandesa para la Comunicación Científica, Universidades de Noruega, y Acción COST Red Europea para la Evaluación de la Investigación en Ciencias Sociales y Humanidades, 2019). Pero también es inevitable pensar en la calidad de la edición y en las causas por las que muchas editoriales apenas son identificadas como prestigiosas. Este estudio, que está relacionado con el reconocimiento de la edición académica de libros en español, pretende subrayar la importancia de considerar la diversidad en la edición "nacional" vinculada con asuntos relevantes en el entorno cercano, así como también la necesidad de cuidar al máximo la calidad y los procesos editoriales para que los libros de la región sean tan reconocidos como aquellos que publican las editoriales del ámbito anglosajón, a menudo multinacionales poco preocupadas por los temas de investigación que no tienen un perfil internacional, y que, además, tienen una posición en el mercado con la que los sellos editoriales nacionales no pueden competir. Estas ideas han impulsado el proyecto Academic Book Publishers: A Global and Interactive Register (Giménez Toledo y Sivertsen, 2019), en el que las editoriales de América Latina, España y Portugal deben estar muy presentes.

Los resultados hacen reflexionar sobre la función de las colecciones en las editoriales académicas colombianas. El hecho de que no pueda hacerse un análisis acerca de las colecciones a partir de las respuestas recibidas, dada la escasez y la dispersión de la información en este sentido, lleva a preguntarse si la noción de colección es funcional en términos de prestigio para los investigadores colombianos, y si las editoriales han sido consistentes en mantener y organizar sus fondos según colecciones claramente reconocibles para el lector.

Considerando los resultados de la tabla 1, las editoriales colombianas más prestigiosas son, en la mayor parte de los casos, editoriales universitarias mientras que en el caso de las editoriales no colombianas, la frecuencia de editoriales universitarias entre las más prestigiosas es significativamente más baja. Este dato está relacionado con la propia conformación del sector editorial académico en Colombia. Lo anterior es congruente con los porcentajes más bajos de editoriales universitarias entre las editoriales no colombianas $(21,45 \%)$ frente al $35,93 \%$ en el caso de las editoriales colombianas. Estos datos apuntan a un mayor peso o reconocimiento de la edición universitaria frente a la edición privada/comercial en Colombia.

Las diferencias ente el prestigio percibido en función de si las respuestas proceden de autores o no autores muestran que el hecho de ser autor no siempre implica una mejor valoración de las editoriales en las que se ha publicado. Esta creencia ha sido en ocasiones utilizada como limitación metodológica a los estudios sobre prestigio de las editoriales. Sin embargo, la propia experiencia del autor con la editorial, en el proceso de edición y publicación, es variable — no siempre es positiva - y eso se aprecia en los resultados. 
Se apunta la existencia de dos polos lingüísticos y culturales de referencia para la academia colombiana, en lo que a edición de libros se refiere: el español/iberoamericano, representado fundamentalmente por España, México y Argentina y el inglés/anglosajón representado por Estados Unidos, Reino Unido y Holanda. A ese análisis hay que añadir que, tomando en consideración el total de editoriales citadas, 81 son latinoamericanas (se calcula que hay unas 400 editoriales universitarias en América Latina) frente a 128 españolas. La circulación de libros, en el caso particular de los académicos, carece de reflexión y análisis. En la cadena del libro hay déficit de distribuidores interesados, libreros formados y editores universitarios que tienen el reto de encontrar rutas más eficientes para difundir y circular su producción. Es innegable que la edición académica latinoamericana viene ganando espacios vía las ferias internacionales del libro, tales como: Guadalajara, Bogotá, Lima o Buenos Aires. Sin embargo, es mucho lo que falta por analizar y desarrollar. Un caso particular, que es elocuente y señala que cuesta optimizar los recursos para la circulación en la región es la falta de transformación digital de las editoriales (Giménez Toledo y Córdoba Restrepo, 2018).

El análisis de los elementos que contribuyen al prestigio percibido de las editoriales señala cuatro aspectos como los más relevantes: calidad científica de los libros publicados, el catálogo de la editorial y su trayectoria, la especialización y el aval científico que suponen los editores. Todos ellos podrían resumirse bajo la etiqueta de "calidad científica", siendo posible concluir, por tanto, que la calidad de los contenidos y el prestigio percibido están estrechamente unidos.

Como apunte final, cabe señalar que la realización de estos estudios a nivel nacional permite disponer de información precisa y contextual que apoye los procesos de evaluación científica, favoreciendo el reconocimiento de la mejor edición académica en español o en otros idiomas distintos al inglés. Construir estas fuentes de información constituye, desde nuestro punto de vista, un impulso para el diseño de modelos de evaluación nacionales y regionales, tal y como el que están impulsando el Consejo Latinoamericano de Ciencias Sociales (Clacso) y el Consejo Nacional de Ciencia y Tecnología (2019), a través de su Primer Foro Latinoamericano sobre Evaluación Científica. Permite, además, una mayor visibilidad de las editoriales de la región en infraestructuras de información internacionales como Academic Book Publishers.

\section{Referencias}

Agencia Nacional de Evaluación de la Calidad y Acreditación (ANECA). (2018). Méritos evaluables para la acreditación nacional para el acceso a los cuerpos docentes universitarios: Catedrático de Universidad. Ciencias sociales y Juridicas. http://www.aneca.es/content/download/15232/187693/file/Criterios\%20Academia\%202 020_ssji_corregido.pdf

Asociación de Editoriales Universitarias de Colombia (ASEUC) (Ed.). (2019). Unilibros de Colombia, 26. http://ase uc.org.co/unilibros/uflip/Unilibros-de-Colombia-26-2019/page_22.html

Centro Regional para el Fomento del Libro en América Latina y el Caribe. (2019). El espacio iberoamericano del libro 2018. Autor. https://cerlalc.org/wp-content/uploads/2019/04/EIL2018_2.pdf

Colciencias. (2012). Convocatoria abierta mediante la cualse invita a las editoriales interesadas en participar en el proceso de registro de editoriales nacionales año 2012 [N. 579]. Departamento Administrativo de Ciencia, Tecnología e Innovación. https://legadoweb.minciencias.gov.co/convocatoria/convocatoria-abierta-mediante-la-cual-se-in vita-las-editoriales-interesadas-en-particip

Colciencias. (2013). Convocatoria para reconocimiento de editoriales nacionales [N. ${ }^{\circ}$ 639]. Departamento Administrativo de Ciencia, Tecnología e Innovación. https://legadoweb.minciencias.gov.co/convocatoria/conv ocatoria-abierta-para-el-reconocimiento-de-editoriales-nacionales-2013

Consejo Latinoamericano de Ciencias Sociales, y Consejo Nacional de Ciencia y Tecnología. (2019, noviembre). Primer Foro Latinoamericano sobre Evaluación Cientifica. México. https://www.clacso.org/foro-latinoamerican o-sobre-evaluacion-cientifica/ 
España. Resolución de 2019. Comisión Nacional Evaluadora de la Actividad Investigadora (CNEAI) [Ministerio de Ciencia, Innovación y Universidades]. Por la que se publican los criterios específicos aprobados para cada uno de los campos de evaluación. Noviembre 12 de 2019. BOE-A-2019-17008.

European Network for Research Evaluation in the Social Sciences and the Humanities, European Cooperation in Science and Technology COST Action 15137 (ENRESSH). (2017, enero). Desafíos de la evaluación de la investigación en Ciencias Sociales y Humanidades. Praga. https://enressh.eu/wp-content/uploads/2017/09/Gu idelines_SSH_Spanish.pdf

Federación de Asociaciones Científicas Finlandesas, Comité para la Información Pública, Asociación Finlandesa para la Comunicación Científica, Universidades de Noruega, y Acción COST Red Europea para la Evaluación de la Investigación en Ciencias Sociales y Humanidades. (2019). Iniciativa Helsinki sobre multilingüismo en la comunicación cientifica. Autores. https://doi.org/10.6084/m9.figshare.7887059

Giménez Toledo, E. (Ed.). (2017) La edición académica española. Indicadores y características (Informe). Federación de Gremios de Editores de España. http://ilia.cchs.csic.es/SPI/spi-fgee/docs/EAEV1.pdf

Giménez Toledo, E. (Ed.). (2018). Estudio cualitativo de las editoriales académicas. La percepción de la comunidad cientifica española. Federación de Gremios de Editores de España. http://ilia.cchs.csic.es/SPI/spi-fgee/docs/EA EV3.pdf

Giménez Toledo, E. (Ed.). (2019). La edición universitaria española. Análisis de la producción editorial de libros. Unión de Editoriales Universitarias Españolas. http://www.une.es/media/Ou1/Image2/webnoviembre2019/Estudio UNE-Produccion_editorial.pdf

Giménez Toledo, E., y Córdoba Restrepo, J. (Eds.). (2018). Edición académica y difusión. Libro abierto en Iberoamérica. Editorial Universidad del Rosario/Comares.

Giménez Toledo, E., y Sivertsen, G. (2019). Academic Book Publishers: A global and interactive register. European Network for Research Evaluation in the Social Sciences and the Humanities. https://enressh.eu/wp-content/u ploads/2019/08/Academic-Book-Publishers-ABP-A-global-and-interactive-register.pdf

Giménez-Toledo, E., Tejada-Artigas, C., y Mañana-Rodríguez, J. (2012). Evaluation of scientific books' publishers in social sciences and humanities: Results of a survey. Research Evaluation, 22(1), 64-77. https://doi.org/10.1093 /reseval/rvs036

Leydesdorff, L., y Felt, U. (2012). Edited volumes, monographs, and book chapters in the Book Citation Index (BKCI) and Science Citation Index (SCI, SoSCI, A\&HCI). Journal of Scientometric Research, I(1). https://doi.org/10 $.5530 /$ jscires.2012.1.7

Scholarly Publishers Indicators Colombia (SPI Colombia). (2019). Editoriales más prestigiosas según los investigadores colombianos. Tableau Public. https://public.tableau.com/profile/.lia\#!/vizhome/SPICol_/Dashboard2

Scholarly Publishers Indicators Expanded (SPI) (2018). http://ilia.cchs.csic.es/SPI/expanded_index.html

Uribe Tirado, A. (2019). Posibilidades de categorización de editoriales según su visibilidad e impacto. Una necesidad para la comunicación científica en Colombia e Iberoamérica. Propuesta metodológica. En M. Acero Gómez (Coord.), Sistemas de evaluación y edición universitaria (pp. 163-190). Asociación de Editoriales Universitarias de Colombia.

\section{Anexos}

\section{Anexo 1. Carta a los académicos colombianos}

Bogotá/Madrid, 24 de octubre de 2018

Estimado/a investigador/a:

El Grupo de Investigación sobre el Libro Académico (ILIA) del Consejo Superior de Investigaciones Científicas (CSIC) de España viene trabajando desde 2012 en el desarrollo de un sistema público de indicadores para editoriales de libros académicos denominado SPI (Scholarly Publishers Indicators) ${ }^{1}$. Esos 
indicadores pretenden ofrecer información objetiva sobre las editoriales académicas a evaluadores de la actividad investigadora, de forma que puedan constituir una ayuda en sus procesos de toma de decisiones.

Tanto desde ASEUC (Asociación de Editoriales Universitarias de Colombia) como desde ILIA consideramos que realizando este tipo de estudios se puede lograr un mejor reconocimiento de la literatura científica publicada en libros en español y en otras lenguas de la región, favoreciendo además su visibilidad y su fortalecimiento.

En el marco de un proyecto de investigación del CSIC y en colaboración con ASEUC, iniciamos en este momento una consulta a profesores e investigadores de universidades colombianas sobre la calidad y el prestigio de las editoriales académicas. Sin duda, su opinión como lectores y autores en una disciplina constituye una base fundamental en la valoración de las editoriales académicas. Por ello, le invitamos a participar en esta consulta, cumplimentando el cuestionario que encontrará en https://docs.google.com/for ms/d/e/1FAIpQLSfa2UFPx_yNBFv6t4ilZ91SYSx-VPDVclcM2ZKdidwZey2oTQ/viewform y que estará disponible hasta el 15 de marzo de 2019. El tiempo estimado para diligenciarlo es de 10 minutos.

La encuesta es anónima y los datos serán tratados de forma agregada. Los resultados de la consulta serán publicados en un informe que les haremos llegar tan pronto esté disponible, así como a través del sitio web SPI.

Esperamos poder contar con su colaboración. No dude en consultarnos las dudas que pudieran surgirle en la dirección de correo XXXXX

Un cordial saludo,

XXXXXXXXXXXXX

\section{Anexo 2. Cuestionario sobre calidad y prestigio de las editoriales}

\section{Perfil del investigador}

1. Por favor, indique su campo específico

2. Por favor, indique su campo detallado

3. Señale el organismo en el que trabaja

II Editoriales académicas colombianas

Señale la editorial académica colombiana que, a su juicio, sea la más prestigiosa en su campo de conocimiento

Por favor, señale si ha publicado en alguna ocasión con la editorial que acaba de nombrar

(Las dos preguntas anteriores se repiten en el cuestionario para cada una de las 10 editoriales que pueden ser nombradas, en orden decreciente de prestigio)

\section{Colecciones académicas}

Señale la colección académica colombiana que, a su juicio, sea la más prestigiosa en su campo de conocimiento

Indique a qué editorial pertenece la colección señalada en la pregunta inmediatamente anterior

(Las dos preguntas anteriores se repiten en el cuestionario para cada una de las 5 colecciones que pueden ser nombradas, en orden decreciente de prestigio)

IV Editoriales académicas extranjeras

Señale la editorial académica extranjera que, a su juicio, sea la más prestigiosa en su campo de conocimiento

Por favor, señale si ha publicado en alguna ocasión con la editorial que acaba de nombrar

(Las dos preguntas anteriores se repiten en el cuestionario para cada una de las 10 editoriales que pueden ser nombradas, en orden decreciente de prestigio)

V Elementos que conforman el prestigio de una editorial

$\mathrm{Al}$ seleccionar las editoriales más prestigiosas ¿qué elementos han intervenido en su decisión? (puede señalar más de una opción) 
La especialización de la editorial

La calidad científica de los libros que publica

La calidad técnica de las obras (diseño editorial, corrección, maquetación, índices, etc.)

Su trayectoria y el fondo editorial que ha creado

El enfoque de los libros que publica, fruto de la visión del/a editor/a

El aval científico que representan los editores y/o directores de colección

El rigor en la selección de originales que se da en la editorial

Los premios que ha recibido la editorial

Su propia experiencia como autor en relación con la eficiencia y competencia del editor en el proceso de publicación

Las recomendaciones de colegas

El prestigio de la editorial entre colegas

Su eficiencia en los procedimientos de información, indexación y de marketing

El hecho de que sus obras sean traducidas a otros idiomas

Las reseñas que suelen recibir sus obras

Otros. Por favor, indique cuáles

\section{Notas}

* El reconocimiento de la edición académica en español. Estudios de caso de Colombia y España.

1 http://ilia.cchs.csic.es/SPI

Licencia Creative Commons CC BY 4.0

Cómo citar este artículo: Giménez Toledo, E., Córdoba Restrepo, J. F., Giraldo González, E., y MañanaRodríguez, J. (2021). Calidad y prestigio de la edición académica. El caso colombiano. Signo y Pensamiento, 40(78). https://doi.org/10.11144/Javeriana.syp40-78.cpea 